\title{
ESTRATÉGIA SAÚDE DA FAMÍLIA: SATISFACÃO DOS USUÁRIOS NO MUNICÍPIO DE FORTALEZA-CE
}

\section{Cesário Rui Callou Filho}

Fisioterapeuta. Mestre em Saúde Coletiva e Professor Líder do Grupo de Estudo em Saúde Coletiva (GESC), Brasil.

\section{Maria de Fátima Antero Sousa Machado}

Doutora em Enfermagem. Docente do Departamento de Enfermagem e do Programa de Pós-Graduação em Enfermagem da Universidade Regional do Cariri, Crato (CE), Brasil.

E-mail: fatimaantero@uol.com.br

\section{Caroline Antero Machado Mesquita}

Cirurgiã-dentista. Mestre em Saúde Coletiva. Professora Líder do Grupo de Estudo em Saúde Coletiva (GESC), Brasil.

\section{Lydiane Koppes Rocha}

Discente da Faculdade Mauricio de Nassau, Fortaleza (CE), Brasil.

\section{Maria Valdilene dos Santos Rodrigues}

Discente da Faculdade Mauricio de Nassau, Fortaleza (CE), Brasil.
RESUMO: O nível de satisfação ou insatisfação pode transcorrer das relações interpessoais entre o profissional e o paciente e, ao mesmo tempo, pode estar relacionado a aspectos da infraestrutura dos serviços e também às representações do usuário sobre o processo saúde-doença. $\mathrm{O}$ estudo tem como objetivo conhecer a satisfação dos usuários quanto ao atendimento na Estratégia Saúde da Família (ESF). Trata-se de um estudo transversal quantitativo, cuja amostra de usuários independe do sexo e faixa etária e exige o cadastro na UBS do estudo. Participaram da pesquisa 301 usuários, dos quais $76,1 \%$ eram do sexo feminino e $23,9 \%$ do sexo masculino. Dentre esses, verificouse que $82,1 \%$ dizem-se satisfeitos com o atendimento prestado pelos profissionais da Estratégia Saúde da Família. Constatou-se um bom grau de satisfação dos usuários da ESF em algumas áreas estudadas, sendo essa satisfação maior quanto à infraestrutura da UBS e ao bom atendimento dos funcionários.

PALAVRAS-CHAVE: Atenção Primária à Saúde; Saúde da Família; Estratégia Saúde da Família; Satisfação do Paciente.

\section{FAMILY HEALTH STRATEGY: USER SATISFACTION IN FORTALEZA CITY}

ABSTRACT: The level of satisfaction or dissatisfaction can be consequence of interpersonal relationships between the professional and the patient, and, at the same time, can be related to the aspects of the material infrastructure of services, in addition to the user representations about the health-disease process. This study aims to identify the main reasons for user satisfaction regarding the service in Family Health Strategy (FHS) program. This is a quantitative and cross-sectional study, whose sample was composed of users enrolled in the PCU under study, regardless of gender and age. The research was made with 301 users, of which $76.1 \%$ were female and $23.9 \%$ were male. Among them, it was observed that $82.1 \%$ say to be satisfied with the service provided by professionals working in Family Health Strategy. It was noted that there was a good level of satisfaction by FHS users in some studied areas, and this satisfaction was greater as the BHU infrastructure and the good services provided by employees.

KEY WORDS: Primary Health Care; Family Health; Family Health Strategy; Patient Satisfaction. 


\section{INTRODUÇÃO}

O Programa de Agentes Comunitários de Saúde (PACS) e a Estratégia Saúde da Família (ESF) foram implementados em grande parte do território brasileiro com a finalidade de estender as ações básicas de saúde para a parte da população que não tinha acesso a esses serviços (FORTES et al., 2014).

Igualmente, possibilitam o envolvimento com a comunidade, especialmente pela atuação dos Agentes Comunitários de Saúde (ACS) e situam as equipes multiprofissionais mais próximas às pessoas em seus espaços de vida (VIDAL et al., 2014).

O ambiente no qual o cuidado de saúde é fornecido deve conter vizinhança e arredores limpos, mobília adaptada, comida saudável e de boa procedência, ventilação satisfatória, água limpa, banheiros e roupas limpos. Devem ser realizados procedimentos regulares de limpeza e conservação da edificação e alicerces da Unidade de Saúde (OMS, 2000).

Os fatores adjuntos ao uso de serviços de saúde e sua satisfação com eles envolvem características sociodemográficas, costumes relacionados à saúde e indicadores das condições de saúde. $\mathrm{O}$ uso desses serviços aumenta com a idade em razão do aumento da incidência e da prevalência de doenças crônicas e insuficiências (LIMA-COSTA; LOYOLA FILHO, 2008).

$O$ nível de satisfação ou insatisfação pode transcorrer das relações interpessoais entre o profissional e o paciente e, ao mesmo tempo, pode estar relacionado a aspectos da infraestrutura, material dos serviços e também com as representações do usuário sobre o processo saúde-doença (SILVA; FORMIGLI, 1994).

Como parte inerente à avaliação dos serviços de saúde voltada para a melhoria da qualidade dos serviços, a satisfação dos usuários permite aperfeiçoar os processos de organização dos serviços, o planejamento em saúde, os processos decisórios em nível de gestão, como também dar um novo significado às proposições de políticas públicas de saúde para os diversos níveis de atenção, além de retroalimentar os processos de trabalho em saúde (ARRUDA; BOSI, 2017).

O objetivo do presente estudo foi reconhecer a satisfação dos usuários quanto ao atendimento na Estratégia Saúde da Família.

\section{METODOLOGIA}

Tratou-se de um estudo transversal, com abordagem quantitativa, realizado em 02 Unidades Básicas de Saúde (UBS) que compõem a Secretaria Executiva Regional (SER) VI do município de Fortaleza, estado do Ceará. Essa Regional atende diretamente a moradores de 29 bairros e cobre $42 \%$ do território de Fortaleza. Foram selecionadas dessa Regional as UBS de maior e de menor porte em relação ao número de atendimentos.

Participaram do estudo usuários com idade acima de 18 anos que estiveram presentes na UBS no momento da coleta de dados, independente do sexo, e que estivessem cadastrados na unidade. Os dados foram coletados entre os meses de junho e setembro de 2015.

A coleta foi realizada por meio de questionário elaborado pelos pesquisadores com base em estudos - DIAS et al, 2011, ESPERIDIÃO, TRAD, 2006 e continham questões relativas ao perfil socioeconômico (idade, sexo, escolaridade, renda familiar), observações sobre a infraestrutura (limpeza do ambiente, salas de atendimento, segurança, estrutura da unidade e outros) e sobre a satisfação dos usuários quanto ao atendimento prestado pelos profissionais da ESF.

Para assegurar a seleção aleatória dos usuários em cada turno da abordagem, foi escolhida randomicamente a hora para o início das entrevistas. O horário de início teve igual probabilidade de ocorrência em períodos de 15 minutos, a começar no início do turno, tendo em vista que os questionários são marcados a cada 15 minutos.

A fim de investigar a satisfação dos usuários, as amostras foram obtidas utilizando uma análise descritiva (univariada), sendo calculadas as frequências absoluta e relativa para todas as variáveis categóricas. Já para as variáveis numéricas foram calculados média, mediana, desvio padrão, mínimo e máximo. Alguns gráficos foram gerados para facilitar a visualização de algumas frequências e uma análise de comparações (bivariada) utilizada para verificar o objetivo apresentado de acordo com a característica das variáveis envolvidas. Dois testes foram utilizados para verificar os objetivos apresentados de acordo com a característica das variáveis: teste de Mann-Whitney (utilizado nas comparações de grupo com apenas duas categorias quanto a uma variável numérica 
e uma alternativa não paramétrica ao teste T de Student para comparação de dois grupos) e teste qui-quadrado (utilizado para verificar a relação significante entre o comportamento de duas variáveis categóricas). O teste exato de Fisher é uma alternativa ao teste qui-quadrado, sendo também utilizado para comparações envolvendo duas variáveis categóricas. Ele substitui o teste quiquadrado quando a disposição dos dados dentro da tabela de contingência apresenta valores esperados baixos (usualmente usa-se como ponto de corte o valor 5). Para o teste de Mann-Whitney e o teste qui-quadrado, foi usado teste de significância de 0,05 .

O projeto foi aprovado pelo Comitê de Ética em Pesquisa (CEP) do Hospital Geral de Fortaleza, com o número de registro 1.422 .794 .

\section{RESULTADOS}

Participaram da pesquisa 301 usuários, dos quais $76,1 \%(n=229)$ eram do sexo feminino e $23,9 \%$ $(\mathrm{n}=72)$ do sexo masculino. Quanto à escolaridade, $48,8 \%$ $(\mathrm{n}=147)$ afirmaram ter ensino médio completo, 48,2\% $(n=145)$ autodeclararam-se solteiros, 35,9\% $(n=108)$ recebem entre 1e 3 salários mínimos e 47,2\% ( $n=142)$ pertencem à religião católica. Quanto à idade, as faixas etárias com maior frequência foram de 18 a 30 anos e 31 a 45 anos, ambas com 36,5\% $(n=110)$. As informações detalhadas do perfil sociodemográfico dos usuários podem ser encontradas na Tabela 1 .

Tabela 1. Caracterização do perfil sociodemográfico dos usuários - Fortaleza - CE (2015) (continua)

\begin{tabular}{|c|c|c|c|c|}
\hline \multirow{2}{*}{ Variável } & Satisfeito & Insatisfeito & \multirow{2}{*}{ IC $95 \%$} & \multirow{2}{*}{ Valor 1} \\
\hline & \multicolumn{2}{|c|}{ N (\%) } & & \\
\hline \multicolumn{5}{|l|}{ Idade } \\
\hline $\begin{array}{l}18 \text { a } 30 \\
\text { anos }\end{array}$ & $86(78,9)$ & $23(21,1)$ & 1,00 & \\
\hline $\begin{array}{l}31 \mathrm{a} 45 \\
\text { anos }\end{array}$ & $93(85,3)$ & $16(14,7)$ & $1,55(0,77-3,14)$ & $0,579 * *$ \\
\hline $\begin{array}{l}46 \text { a } 65 \\
\text { anos }\end{array}$ & $46(82,1)$ & $10(17,9)$ & $1,23(0,54-2,81)$ & \\
\hline 65 ou mais & $22(88,0)$ & $03(12,0)$ & $1,96(0,54-7,13)$ & \\
\hline
\end{tabular}

\begin{tabular}{|c|c|c|c|c|}
\hline \multirow{2}{*}{ Variável } & Satisfeito & Insatisfeito & \multirow{2}{*}{ IC 95\% } & \multirow{2}{*}{ Valor $p$} \\
\hline & \multicolumn{2}{|c|}{ N (\%) } & & \\
\hline Casado & $94(81,0)$ & $22(19,0)$ & $3,2(0,67-15,36)$ & $0,159 * *$ \\
\hline Solteiro & $123(85,4)$ & $21(14,6)$ & $4,39(0,92-21,05)$ & \\
\hline Viúvo & $13(92,9)$ & $01(7,1)$ & $9,75(0,78-121,84)$ & \\
\hline Divorciado & $10(71,4)$ & $04(28,6)$ & $1,88(0,28-12,46)$ & \\
\hline $\begin{array}{l}\text { União } \\
\text { estável }\end{array}$ & $04(57,1)$ & $03(42,9)$ & 1,00 & \\
\hline \multicolumn{5}{|l|}{ Religião } \\
\hline Católico & $119(84,4)$ & $22(15,6)$ & $2,70(0,84-8,68)$ & $0,460 * *$ \\
\hline Evangélico & $105(84,0)$ & $20(16,0)$ & $2,63(0,81-8,5)$ & \\
\hline Espírita & $01(100,0)$ & - & - & \\
\hline Outra & $09(90,0)$ & $01(10,0)$ & $4,50(0,44-46,17)$ & \\
\hline Ateu & $10(66,7)$ & $05(33,3)$ & 1,00 & \\
\hline \multicolumn{5}{|l|}{ Sexo } \\
\hline Masculino & $59(83,1)$ & $12(16,9)$ & $1,05(0,52-2,12)$ & $0,901^{*}$ \\
\hline Feminino & $188(82,5)$ & $40(17,5)$ & 1,00 & \\
\hline \multicolumn{5}{|l|}{ Escolaridade } \\
\hline $\begin{array}{l}\text { Não } \\
\text { estudou }\end{array}$ & $02(66,7)$ & $01(33,3)$ & 1,00 & \\
\hline $1^{\mathrm{a}}-4^{\mathrm{a}}$ série & $27(96,4)$ & $01(3,6)$ & $13,5(0,59-306,31)$ & $0,073 * *$ \\
\hline $5^{\mathrm{a}} \cdot 8^{\mathrm{a}}$ série & $74(87,1)$ & $11(12,9)$ & $3,36(0,28-40,27)$ & \\
\hline $\begin{array}{l}\text { Ensino } \\
\text { médio }\end{array}$ & $118(81,4)$ & $27(18,6)$ & $2,19(0,19$ - 24,99) & \\
\hline $\begin{array}{l}\text { Ensino } \\
\text { superior }\end{array}$ & $26(74,3)$ & $09(25,7)$ & $1,44(0,12-17,9)$ & \\
\hline
\end{tabular}

Renda

mensal

\begin{tabular}{lllll}
\hline $\begin{array}{l}\text { Nenhuma } \\
\text { renda }\end{array}$ & $19(79,2)$ & $05(20,8)$ & $1,14(0,22-5,78)$ & $0,781^{* *}$ \\
Até 1 SM & $130(83,9)$ & $25(16,1)$ & $1,56(0,40-6,07)$ & \\
$\begin{array}{l}\text { De 1 a 3 } \\
\text { SM }\end{array}$ & $88(83,0)$ & $18(17,0)$ & $1,47(0,37-5,87)$ \\
$\begin{array}{l}\text { Mais de } \\
\text { 3SM }\end{array}$ & $10(76,9)$ & $03(23,1)$ & 1,00 \\
\hline
\end{tabular}

Reside

(tempo)

\begin{tabular}{lcccc}
\hline $\begin{array}{l}\text { 5 a } 10 \text { anos } \\
\text { 31 (77,5) }\end{array}$ & $09(22,5)$ & $1,38(0,46-4,16)$ & $0,204^{* *}$ \\
$\begin{array}{l}10 \text { a } 15 \\
\text { anos }\end{array}$ & $46(88,5)$ & $06(11,5)$ & $3,07(0,94-10,0)$ \\
$\begin{array}{l}15 \text { a } 20 \\
\text { anos }\end{array}$ & $42(89,4)$ & $05(10,6)$ & $3,36(0,97-11,58)$ \\
$\begin{array}{l}\text { 20 ou mais } \\
\text { anos }\end{array}$ & $107(81,7)$ & $24(18,3)$ & $1,78(0,70-4,53)$ \\
1 a 5 anos & $20(71,4)$ & $08(28,6)$ & 1,00 \\
\hline *Teste de Qui-quadrado & **Teste Extrato de Fisher
\end{tabular}




\subsection{SATISFAÇÃO DOS USUÁRIOS EM RELAÇÃO À INFRA-} ESTRUTURA

Verificou-se que $44,2 \% \quad(n=133) \quad$ dos entrevistados residem há mais de 20 anos na área atendida pela ESF. Com relação à utilização do serviço, $56,5 \%(n=170)$ afirmaram que utilizam os serviços da UBS com frequência. Destes, 56,5\% $(n=170)$ consideram que a Unidade Básica de Saúde tem uma infraestrutura adequada e $69,1 \%(n=208)$ dos participantes alegaram que precisam enfrentar filas para serem atendidos.

Dos entrevistados, $41,9 \%(n=126)$ classificaram $\mathrm{o}$ atendimento prestado pela recepção como sendo bom.
Referente à segurança na UBS, 36,9\% (n=111) dos usuários classificaram-na como péssima. Na questão de limpeza da UBS, 48,8\% $(n=147)$ consideram-na boa.

Sobre a estrutura física das salas da UBS, a sala de preparo foi bem avaliada pelos usuários, 50,2\% $(n=151)$ consideram a estrutura boa. Já em relação à farmácia, 31,9\% $(n=96)$ dos sujeitos consideramna razoável, 39,5\% $(n=119)$ consideram boa a sala de enfermagem, $41,5 \%(n=125)$ acham boa a estrutura dos consultórios médicos, $18,9 \%(n=57)$ consideram boa a sala de curativo e, finalmente, 54,2\% $(n=163)$ avaliaram como boa a estrutura física da sala de vacina. Informações detalhadas podem ser vistas na Tabela 2 .

Tabela 2. Descrição das características da infraestrutura da UBS de acordo com os usuários - Fortaleza-CE (2015)

\begin{tabular}{|c|c|c|c|c|c|c|c|c|c|}
\hline \multirow{2}{*}{$\begin{array}{c}\text { Avaliação dos } \\
\text { quesitos }\end{array}$} & \multicolumn{4}{|c|}{ Satisfeitos } & \multicolumn{4}{|c|}{ Insatisfeitos } & \multirow[b]{2}{*}{ Valor $p$} \\
\hline & $\mathbf{N}$ & Média & Mediana & $\begin{array}{l}\text { Desvio } \\
\text { Padrão }\end{array}$ & $\mathbf{N}$ & Média & Mediana & $\begin{array}{l}\text { Desvio } \\
\text { Padrão }\end{array}$ & \\
\hline Limpeza & 247 & 4,316 & 5 & 0,9181 & 52 & 3,827 & 4 & 1,1328 & 0,002 \\
\hline Assiduidade & 247 & 3,996 & 4 & 1,0221 & 51 & 3,118 & 3 & 1,3949 & $<0,001$ \\
\hline Segurança & 246 & 3,358 & 3 & 1,3256 & 50 & 2,9 & 3 & 1,374 & 0,026 \\
\hline UBS & 247 & 3,903 & 4 & 1,1435 & 51 & 3,451 & 4 & 1,238 & 0,013 \\
\hline
\end{tabular}

Teste de Mann Whitney

3.2 SATISFAÇÃO DOS USUÁRIOS EM RELAÇÃO AO TRABALHO DOS PROFISSIONAIS DA ESF

Outro aspecto pesquisado foi a aceitação dos profissionais da Estratégia Saúde da Família (ESF), atribuindo-se uma nota de 0 a 10 pontos, em que $82,1 \%$ $(n=247)$ dos entrevistados dizem-se satisfeitos com o atendimento prestado por esses profissionais e apenas
$17,3 \%(n=52)$ não estão satisfeitos. Neste estudo, a maior média foi atribuída ao médico, 8,87. Os auxiliares de enfermagem tiveram uma média de 8,26 , os enfermeiros de 8,22 e o dentista teve a média mais baixa, 5,8. Os ACS obtiveram uma média de 6,59, o Núcleo de Atendimento ao Cliente (NAC) obteve média de 7,95 e o gestor da unidade teve média 7,8. Informações detalhadas podem ser vistas na Tabela 3.

Tabela 3. Distribuição das notas atribuídas aos profissionais da Estratégia Saúde da Família em relação ao atendimento prestado por esses profissionais - Fortaleza-CE (2015)

\begin{tabular}{|c|c|c|c|c|c|c|c|c|c|}
\hline \multirow[b]{2}{*}{ Avaliação dos profissionais } & \multicolumn{4}{|c|}{ Satisfeitos } & \multicolumn{4}{|c|}{ Insatisfeitos } & \multirow[b]{2}{*}{ Valor $\mathbf{p}$} \\
\hline & $\mathbf{N}$ & Média & Mediana & $\begin{array}{l}\text { Desvio } \\
\text { Padrão }\end{array}$ & $\mathbf{N}$ & Média & Mediana & $\begin{array}{l}\text { Desvio } \\
\text { Padrão }\end{array}$ & \\
\hline Médico & 246 & 8,87 & 10,0 & 1,7 & 52 & 6,64 & 7,5 & 3,2 & $<0,001$ \\
\hline Enfermeiro & 246 & 8,22 & 8,0 & 1,8 & 51 & 5,88 & 6 & 2,7 & $<0,001$ \\
\hline Auxiliar de enfermagem & 244 & 8,26 & 8,5 & 1,8 & 51 & 6,59 & 7 & 3,0 & $<0,001$ \\
\hline Dentista & 156 & 5,8 & 6,0 & 2,9 & 32 & 3,66 & 3 & 2,6 & $<0,001$ \\
\hline Gestor & 203 & 7,08 & 7,0 & 2,2 & 44 & 5,18 & 5 & 2,8 & $<0,001$ \\
\hline Agentes & 233 & 6,59 & 7,0 & 3,0 & 47 & 4,36 & 3 & 3,5 & $<0,001$ \\
\hline NAC & 233 & 7,95 & 8,0 & 2,2 & 51 & 5,57 & 5 & 3,0 & $<0,001$ \\
\hline
\end{tabular}

Teste de Mann Whitney 


\section{DISCUSSÃO}

\subsection{CARACTERIZAÇÃO DA POPULAÇÃO}

Pesquisas que abordam a satisfação de usuários ocupa um lugar mais importante na observação da qualidade dos serviços. Essa posição considera que a satisfação está diretamente relacionada à adesão terapêutica e aos resultados com cuidados em saúde, influenciando comportamentos de saúde e doença, além da crescente valorização do papel do usuário enquanto consumidor (ALBUQUERQUE; DEVEZA, 2009).

Corroborando com os estudos de Ibañez et al. (2006) e Trad et al. (2002), a faixa etária desta pesquisa foi semelhante, visto que nessa população a maior parcela, $73 \%$, encontra-se com idade de 18 a 45 anos. Dentre esses, as mulheres correspondem quase à totalidade e foram mais suscetíveis a responder às perguntas, corroborando com outros estudos encontrados na literatura (BRANDÃO; GIOVANELLA; CAMPOS, 2013; COYLE, 1999; KALCKMANN; BATISTA; SOUZA,2005; SCHRAIBER,2005).

$\mathrm{O}$ autor afirma que a maior demanda nos serviços de saúde, em particular na Saúde da Família, é de pessoas do sexo feminino, indicando que as mulheres utilizam os serviços de saúde com mais frequência, além de assumirem a responsabilidade em procurar atendimento para seus familiares e amigos na sua comunidade (BRANDÃO; GIOVANELLA; CAMPOS, 2013). Atrelado a isso, outro estudo aponta que as mulheres estão mais aptas a criticar os serviços, o que as tornam boas informantes para as pesquisas, já que elas estão mais presentes (COYLE, 1999). Diferentemente para o sexo masculino, em que estudos ressaltam o fato dos homens não se identificarem como objetivo do atendimento dos programas de saúde, uma vez que as ações preventivas dirigiam-se quase exclusivamente às mulheres (KALCKMANN; BATISTA; SOUZA, 2005; SCHRAIBER, 2005).

Em relação à faixa etária, que nesta pesquisa foi de adultos/jovens, Brandão et al afirmam que as faixas etárias mais elevadas podem influenciar os resultados de satisfação, uma vez que em seu estudo os adultos estão propensos a estar mais satisfeitos com os serviços do que os mais jovens. Segundo Mishima et al. (2010), para os adultos, a utilização dos serviços pode ter significado a busca da satisfação de outras necessidades, e não apenas a resolução de um problema de saúde, expresso em um agravo ou conjunto de sinais e sintomas, levando, assim, a uma maior satisfação.

$\mathrm{Na}$ Comissão Nacional sobre Determinantes Sociais da Saúde coloca-se que os efeitos no nível de instrução aparecem na forma de percepção dos problemas de saúde, na adoção de estilos de vida saudáveis, no consumo e na utilização de serviços de saúde, bem como na adesão aos procedimentos terapêuticos (COMISSÃO NACIONAL SOBRE DETERMINANTES SOCIAIS DA SAÚDE, 2008). Neste estudo, a maioria dos entrevistados declara ter ensino médio completo, com um percentual de $48,8 \%$, ao contrário de alguns estudos, como o de Cotta et al. (2005), no qual 54,7\% dos entrevistados afirmaram ser analfabetos funcionais, e no estudo de Oliveira et al. (2014), no qual o principal nível de escolaridade foi ensino fundamental incompleto, com 52,4\%. Para alguns autores, a explicação pode estar na instalação das unidades de saúde da família em regióes de baixa renda e, com isso, a escolaridade de usuários da ESF em geral é baixa. Ainda que alguns autores na literatura internacional não encontrem uma relação entre satisfação e renda ou entre satisfação e escolaridade, no Brasil parece haver uma equiparação inversa, estando os usuários de camadas mais populares mais satisfeitos com os serviços (SANTOS, 1995; SOUZA, 1997).

\subsection{CARACTERÍSTICAS DO ESPAÇO FÍSICO DA UNIDA- DE BÁSICA DE SAÚDE (UBS)}

As opiniões sobre satisfação dos serviços públicos podem atuar como mecanismo para dar voz aos usuários, abrindo oportunidades de expressão em que os mesmos possam monitorar e fiscalizar as atividades dos serviços públicos de saúde (ESPERIDIÃO; TRAD, 2006). Aspectos voltados à infraestrutura da UBS comprometem a forma como os usuários percebem a unidade (IBAÑEZ, 2006). O acesso à unidade é um requisito obrigatório para que a atenção primária torne-se porta de entrada para o sistema de saúde, sendo necessária a extinção de barreiras financeiras, geográficas, organizacionais e 
culturais (ALMEIDA; FAUSTO; GIOVANELLA, 2011). Neste estudo, verificou-se que $44,2 \%$ dos entrevistados residem há mais de 20 anos nas proximidades da UBS e, destes, $56,5 \%$ consideram que a ela tem uma infraestrutura adequada.

Para o Ministério da Saúde, a ambiência na saúde diz respeito ao tratamento dado ao espaço físico, que é entendido como um espaço social, profissional e de relações interpessoais, devendo proporcionar uma atenção acolhedora, resolutiva e humana (BRASIL, 2010). No estudo em questão, as variáveis referentes a características da unidade, como segurança, limpeza da unidade esalas de atendimento, tiveram uma boa aceitação por parte dos usuários, que as consideram boas.

\subsection{SATISFAÇÃO DOS USUÁRIOS EM RELAÇÃO AO TRABALHO DOS PROFISSIONAIS DA ESF}

É necessário ressaltar que a qualidade da assistência prestada dentro dos serviços de saúde é imprescindível para o planejamento e gerenciamento dos sistemas de saúde. A mensuração da satisfação do paciente a partir da utilização dos serviços de saúde é um dos componentes dos resultados desejáveis da assistência prestada (FONSECA, GUTIÉRREZ E ADAMI, 2006).

A qualificação relativa aos profissionais da Estratégia Saúde da Família neste estudo foi satisfatória, $82,1 \%$ dos participantes dizem-se satisfeitos com atendimento prestado por esses profissionais e apenas $17,3 \%$ não estão satisfeitos. Pode-se perceber que o médico foi o profissional mais bem avaliado. Por sua vez, o dentista recebeu a nota mais baixa. Isso pode ter acontecido devido à saúde bucal ter sido implantada na Estratégia Saúde da Família do município de Fortaleza há menos tempo que o serviço de medicina. Ainda existe uma grande demanda reprimida por esse tipo de serviço, o que pode justificar avaliação mais baixa.

A qualificação dos serviços de saúde permite oferecer diretrizes e opções para o processo de planejamento, podendo favorecer o controle técnico e social dos serviços prestados à sociedade (IBAÑEZ, 2006).

A comunicação entre os profissionais de saúde e os usuários (clareza das informações, explicação sobre o diagnóstico e tratamento, esclarecimento de dúvidas e disponibilidade para contato em situações emergenciais) tem ganhado relativa autonomia entre as dimensões da satisfação, sendo privilegiada na mensuração da satisfação (RUBIM, 1989; SITZIA; WOOD, 1997; WILLIAMS et al., 1998 apud ESPERIDIAO, M.; TRAD, 2005).

\section{CONCLUSÃO}

Considera-se que os métodos de observação da satisfação dos usuários nos serviços de saúde podem agir para a construção de um novo aspecto do cuidado, além de monitorar as atividades desses serviços, fortalecer o controle social e a participação dos usuários nos processos de planejamento. No presente estudo, o grupo avaliado é composto por indivíduos de classe econômica baixa, majoritariamente mulheres e com nível médio de escolaridade.

Constata-se um bom grau de satisfação dos usuários da Estratégia Saúde da Família em alguns aspectos, tais como infraestrutura da UBS, salas de vacinas, consultórios médicos e salas de enfermagem, limpeza das áreas da unidade, bom atendimento dos funcionários, médicos, auxiliares de enfermagem e recepcionistas.

Essa pesquisa sugere que estudos de satisfação devem verificar o que pode inferir a necessidade de mudanças e melhorias na infraestrutura, que vão desde a presença de unidades de saúde próprias às condições de apoio diagnóstico e terapêutico (equipamentos, medicamentos e insumos básicos), uma vez que os dados apontam uma melhor reorganização do trabalho, na estima de garantir, em cada área, a presença contínua e diária das equipes de saúde da ESF.

Sabendo da complexidade do tema, não se pretende, com esse estudo, esgotar o assunto. Ao fim de uma pesquisa, algumas dúvidas sempre emergem. Algumas fragilidades encontradas podem ser aprofundadas em futuros trabalhos, como minimizar variáveis que podem estar atreladas há aspectos de políticos, ampliar o número de UBS e amostragem e, principalmente, trazer sugestões sobre as fragilidades apontadas. A percepção na ótica de avaliação qualitativa é de grande importância para o processo de gestão/organização em saúde.

Vale destacar ainda a importância da realização de pesquisas com outros atores envolvidos neste processo, como profissionais e gestores, que devem ser escutados para que sejam obtidos resultados mais robustos. 


\section{REFERÊNCIAS}

ALBUQUERQUE, A. B.; DEVEZA, M. Adesão ao tratamento na prática do médico de família e comunidade e na atenção primária à saúde. In:Programa de atualização em medicina de família e comunidade. Porto Alegre: Artmed; 2009. v. 3, p.41-71.

ALMEIDA, P. F.; FAUSTO, M. C. R.; GIOVANELLA, L. Fortalecimento da atenção primária à saúde: estratégia para potencializar a coordenação dos cuidados. Rev. panam. salud pública, Washington,v. 29, n. 2, p. 84-95, fev. 2011.

ARRUDA, C. A. M.; BOSI, M. L. M. User's satisfaction of primary health care: a qualitative study in the Northeast of Brazil. Interface comun. saúde educ., Botucatu, v. 21, n. 61, p. 321-332, abr.jun. 2017.

BRANDÃO, A. L. R. B. S.; GIOVANELLA, L.; CAMPOS, C. E. A. Avaliação da atenção pela perspectiva dos usuários: Adaptação do instrumento EUROPEP para grandes centros urbanos brasileiros. Cienc. saúde coletiva, Rio de Janeiro, v. 18, n. 1, p. 103-114, jan. 2013.

BRASIL. Ministério da Saúde. Secretaria de Atenção à Saúde. Núcleo Técnico da Política Nacional de Humanização. Ambiência. 2. ed. Brasília: Ministério da Saúde, 2010.

COMISSÃO NACIONAL SOBRE DETERMINANTES SOCIAIS DA SAÚDE. As causas sociais das iniquidades em saúde no Brasil. Rio de Janeiro: FIOCRUZ, 2008.

COTTA, R.M. M.; MARQUE, E. S.; MAIA, T. M.; AZEREDO, C. M.; SCHOTT, M.; FRANCESCHINI, S. C. C.; PRIORE, S. E. A satisfação dos usuários do programa de saúde da família: avaliando o cuidado em saúde. Sci. med., Porto Alegre, v. 15, n. 4, P. 227-234, out./dez. 2005.

COYLE, J. Exploring the meaning of "dissatisfaction" with health care: the importance of "personal identity threat". Sociol Health Illn, v. 21, n. 1, p. 95-123, jan. 1999.

DIAS, O. V; VIEIRA, M. A; DIAS, J. P.; RAMOS, L.H. As dimensões da satisfação dos usuários do Programa Saúde da Família: confiabilidade e empatia. Acta paul. enferm, vol.24, n.2, pp.225-231, 2011.
ESPERIDIÃO, M. A.; TRAD, L. A. B. Avaliação da satisfação de usuários: considerações teórico-conceituais. Cad. saúde pública, Rio de Janeiro, v. 22, n. 6, p. 1267-1276, jun. 2006.

ESPERIDIAO, M.; TRAD, L. A. B. Avaliação de satisfação de usuários. Ciênc. saúde coletiva, Rio de Janeiro, v. 10, supl. p. 303-312, dez. 2005.

FONSECA, S.M.; GUTIERREZ, M.G.R.; ADAMI, N.P. Avaliação da satisfação de pacientes oncológicos com atendimento recebido durante o tratamento antineoplásico ambulatorial. Rev. bras. enfermagem, Brasília, v. 59, n. 5, out, 2006.

FORTES, S.; MENEZES, A.; ATHIÉ, K.; CHAZAN, L. F.; ROCHA, H.; THIESEN, J.; RAGONI, C.; PITHON, T.; MACHADO, A. Psiquiatria no século XXI: transformações a partir da integração com a atenção primária pelo matriciamento. Physis, Rio de Janeiro, v. 24, n. 4, p. 1079-1102, out./dez. 2014.

IBAÑEZ, N.; ROCHA, J. S. Y.; CASTRO, P. C.; RIBEIRO, M. C. S. A.; FORSTER, A. C.; NOVAES, M. H. D.; VIANA, A. L. A. Avaliação do desempenho da atenção básica no estado de São Paulo. Cienc. saúde coletiva,Rio de Janeiro, v. 11, n. 3, p. 683-703, jul./set. 2006.

KALCKMANN, S.; BATISTA, L. E.; SOUZA, L. C. F. Homens de baixa renda falam sobre saúde reprodutiva e sexual. In: ADORNO, R.; ALVARENGA, A.; VASCONCELOS, M. P. (Orgs.). Jovens, trajetória, masculinidades e direitos. São Paulo: Edusp, 2005. p. 199-217.

LIMA-COSTA, M. F.; LOYOLA FILHO, A. I. Fatores associados ao uso e à satisfação com os serviços de saúde entre usuários do sistema único de saúde na região metropolitana de Belo Horizonte, estado de Minas Gerais, Brasil. Epidemiol. serv. saúde, Brasília, v. 17, n. 4, p. 247-257, dez. 2008. 
MISHIMA, S. M.; PEREIRA, F. H.; MATUMOTO, S.; FORTUNA, C. M.; PEREIRA, M. J. B.; CAMPOS, A. C.; PAULA, V. G.; DOMINGOS, M. M. L. N. A assistência na saúde da família sob a perspectiva dos usuários. Rev. latinoam. enferm., Ribeirão Preto, v. 18, n. 3, p. 148156, maio/jun. 2010.

OLIVEIRA, L. P. B. A.; MEDEIROS, L. M.F.; MEIRELLES, B. H. S. SANTOS, S. M. A. satisfação da população idosa atendida na estratégia desaúde da família de Santa Cruz, Rio Grande do Norte. Texto \& contexto enferm., Florianópolis, v. 23, n. 4, p. 871-879, out./dez. 2014.

ORGANIZAÇÃO MUNDIAL DA SAÚDE.OMS. Statistical Information System (WHOSIS). 2000.

SANTOS, M. P. Avaliação da qualidade dos serviços públicos de atenção à saúde da criança sob a ótica do usuário. 1995. Dissertação (Mestrado). - Instituto de Saúde Coletiva, Universidade Federal da Bahia, Salvador, 1995.

SCHRAIBER, L. B. Equidade de gênero e saúde: o cotidiano das práticas no programa saúde da família do Recife. In: VILLELA, W.; MONTEIRO, S. (Orgs.). Gênero e saúde: programa saúde da família em questão. Rio de Janeiro: ABRASCO; Brasília: Fundo de População das Nações Unidas, 2005. p. 39-61.

SILVA, L. M. V.; FORMIGLI, V. L. A. Avaliação em saúde: limites e perspectivas. Cad. saúde pública, Rio de Janeiro, v. 10, n. 1, p. 80-91, mar. 1994.

SOUZA, E. M. A satisfação dos idosos com os serviços de saúde: um estudo de prevalência e de fatores associados em Taguatinga, Distrito Federal. 1997. $154 \mathrm{f}$. Dissertação (Mestrado). - Faculdade de Ciências da Saúde, Universidade de Brasília, Brasília, 1997.

TRAD, L. A. B.; BASTOS, A. C. S.; SANTANA, E. M.; NUNES, M. O. Estudo etnográfico da satisfação de usuário do programa de saúde da família (PSF) na Bahia. Cienc. saúde coletiva, Rio de Janeiro, v. 7, n. 3, p. 581-589, 2002.
VIDAL, S. V.; MOTTA, L. C. S.; GOMES, A. P.; SIQUEIRA - BATISTA, R. Problemas bioéticos na estratégia saúde da família: reflexões necessárias. Rev. bioét., Brasília, v. 22, n. 2, p. 347-357, ago. 2014.

Recebido em:29 de maio de 2017 Aceito em: 30 de agosto de 2017 